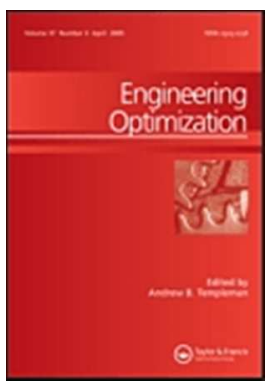

\title{
Level set topology optimization for multimaterial and multifunctional mechanical metamaterials
}

\begin{tabular}{|r|l|}
\hline Journal: & Engineering Optimization \\
\hline Manuscript ID & GENO-2015-0193.R4 \\
\hline Manuscript Type: & Original Article \\
\hline Date Submitted by the Author: & $\mathrm{n} / \mathrm{a}$ \\
\hline Complete List of Authors: & $\begin{array}{l}\text { Wang, Yu; The University of Technology, Sydney, School of Electrical, } \\
\text { Mechanical and Mechatronic Systems } \\
\text { Gao, Jie; Huazhong University of Science and Technology, Mechanical } \\
\text { Engineering } \\
\text { LUO, ZHEN; The University of Technology, Sydney, School of Electrical, } \\
\text { Mechanical and Mechatronic Systems } \\
\text { Brown, Terry; The University of Technology, Sydney, School of Electrical, } \\
\text { Mechanical and Mechatronic Systems } \\
\text { Zhang, Nong; The University of Technology, Sydney, School of Electrical, } \\
\text { Mechanical and Mechatronic Systems }\end{array}$ \\
\hline Keywords: & $\begin{array}{l}\text { Mechanical metamaterials, Topological shape optimization, Multiphase let } \\
\text { set method }\end{array}$ \\
\hline
\end{tabular}

\section{SCHOLARONE ${ }^{\text {N }}$}

Manuscripts 


\title{
Level set topology optimization for multimaterial and multifunctional mechanical metamaterials
}

By

\author{
Yu Wang ${ }^{1}$, Jie Gao ${ }^{2}$, Zhen Luo ${ }^{1, *}$, Terry Brown ${ }^{1}$, Nong Zhang ${ }^{1}$ \\ ${ }^{1}$ School of Electrical, Mechanical and Mechatronic Systems \\ The University of Technology, Sydney, NSW 2007, Australia \\ ${ }^{2}$ State Key Lab of Digital Manufacturing Equipment and Technology \\ Huazhong University of Science and Technology, Wuhan, Hubei 430074, China \\ * Correspondence author: \\ (Dr Z. Luo, Email: zhen.luo@uts.edu.au; Phone: +61 29514 2994; Fax: +61 29514 2655)
}

\begin{abstract}
Metamaterials are artificially engineered composites designed to have unusual properties. This paper will develop a new level set based topology optimization method for computational design of multimaterial metamaterials with exotic thermo-mechanical properties. In order to generate metamaterials consisting of arrays of microstructures under periodicity, the numerical homogenization method is used to evaluate the effective properties of the microstructure, and a multiphase level set model is used to evolve boundaries of the multi-material microstructure. The proposed method will produce material geometries with distinct interfaces and smoothed boundaries, which may facilitate the fabrication of the topologically optimized designs. Several numerical cases are used to demonstrate the effectiveness of the proposed method.
\end{abstract}

Key Words: Mechanical metamaterials; Topological shape optimization; Multiphase level set method. 


\section{Introduction}

Interest in designing new materials that exhibit exotic properties in engineering and science is increasing. In particular, metamaterials, a family of man-made composites with unconventional properties and ubiquitous applications, have recently been experiencing great popularity (Lakes 1987, 1996; Evan and Alderson 2000; Smith, Pendry and Wiltshire 2004). The unusual properties of metamaterials arises from specifically designed and configured micro- and nanostructures rather than from their composition. In other words, the layout of the internal structural geometry plays an important role in determining the properties of metamaterials. Early revolutionary use of metamaterials was demonstrated by Veselage (Veselage 1968), who developed electromagnetic metamaterials exhibiting negative permittivity and permeability. Recently, the concept of electromagnetic metamaterials (Vegelago 1968; Smith, Pendry and Wiltshire 2004) has been applied with different parameters to design mechanical metamaterials. Several different types of mechanical metamaterials have been developed, e.g. acoustic metamaterials (Chen and Chan 2007), negative Poisson's ratio (NPR) metamaterials (known as auxetics) (Lake 1987; Evan and Alderson 2000; Milton 1992), and negative compressibility metamaterials (Nicolaou and Motter 2012).

Poisson's ratio is usually defined as the ratio of transverse contraction strain to longitudinal extension strain under uniaxial tension, and is a fundamental property of elastic materials. In contrast to conventional materials with positive Poisson's ratios, materials with negative Poisson's ratios expand laterally when stretched and contract laterally when compressed. Since the work of (Lakes 1987), NPR metamaterials (auxetics) have attracted much attention. So far, several intuitional and heuristic methods have been developed to design NPR metamaterials (Yang et al. 2004). However, when auxetic materials are subjected to temperature variations, the thermal properties will also cause contraction and extension of structures and hence affect the properties. The thermoelastic property (co-efficient of expansion) is normally positive for most conventional materials. The additional effects of thermal properties on deformation makes the intuitional and heuristic methods even more ineffective and inefficient.

Multifunctional metamaterials with both controlled thermal expansion and deformation have already found, and should continue to find, interesting and novel engineering applications. For example, in aerospace aviation, a deployable antenna for deep-space missions was enhanced by exploiting the unique properties of auxetics (Scarpa and Tomlinson 2000). However, these structures must operate in an extremely cold environment at cryogenic temperatures. Even in such a harsh environment, the deployable devices with auxetics are required to expand and contract as intended. Other applications, such as blast curtains, are required to open small holes when expanded so as to only let the air through but capture all flying shards of different sizes. These curtains, with filtering and protection functions that must operate when subjected to 
large changes in surrounding temperature, can be designed with multifunctional mechanical metamaterials to exploit their auxetic behavior.. Hence, it is desirable to design multifunctional metamaterials with controls of both NPR and zero thermal expansion (ZTE) in these circumstances. Topology optimization can be used to design these mechanical metamaterials with desired multi-functional properties and this is an application seldom studied in the field of topology optimization.

In order to exploit the unique properties of metamaterials in engineering applications it is necessary to design them to have the expected properties. Topology optimization is considered to be a promising tool to achieve this. Numerically, topology optimization (Bensøe and Sigmund 2003) is an iterative process to determine the best structural layout by re-distributing a prescribed amount of material within a fixed reference domain, until the objective function is minimized under constraints. Topology optimization has experienced considerable development leading to several different methods (Bensøe and Kikuchi 1988; Bensøe and Sigmund 1999; Zhou and Rozvany 1991; Xie and Steven 1993; Sethian and Wiegman 2000; Wang, Wang, and Guo 2003; Allaire, Jouve, and Toader 2004; Kang and Wang 2011; Luo et al. 2013). However, a new systematic design method is required for more effectively engineering metamaterials with unusual thermo-mechanical properties. Firstly, a new topological description model is essential for topology optimization of multi-phase metamaterials (Tavakoli and Mohseni 2014) since most existing methods are only applicable to single phase materials. A representation model should indicate each phase in the design domain distinctly without overlap and/or voids and an explicit formulation is required for sensitivity analysis.

A few models for describing multimaterial microstructures have been developed. For instance, Bendsøe and Sigmund (1999) proposed a mixture rule for a multi-material model using the SIMP (Solid Isotropic Material with Penalization) method. Several extended versions of SIMP have been applied to multimaterial designs (Sigmund 2001; Luo et al. 2010; Gao and Zhang 2011). The homogenization method (Bensøe and Kikuchi 1988; Allaire 2002) has also been used in the design of multi-phase composites (Cherkaev 2000; Sigmund and Torquato 1996, 1997; Gibiansky and Sigmund 2000). In addition, the phase field method, representing structural domains and interfaces as a whole by a set of field variables, has been applied to multi-material design problems (Zhou and Wang 2007; Tavakoli 2014). Other multi-material topology optimization models have been developed for multi-component structures (Zhu, Zhang and Beckers 2009; Buehler, Bettig and Parker 2004; Wang and Wang 2004).

Since the work of (Sethian and Wiegmann 2000), several different level set methods (LSMs) (Wang, Wang, and Guo 2003; Allaire, Jouve, and Toader 2004; Wang and Wang 2004; Yamada et al. 2010) within the standard level set framework have been developed, including topology optimization of multi-material structures and metamaterials (Wang and Wang 2004; Mei and Wang 2004; Wang and Wang 2005; Allaire et 
al. 2014; Zhou et al. 2011; Otomori et al. 2012; Lu et al. 2013). Recently, some alternative LSMs in parametric or equivalent forms have been developed to achieve topological shape design of structures (Belytscho, Xiao and Parimi 2003; Haber 2004; Luo et al. 2007; Luo et al. 2008; Luo et al. 2009; Wei and Wang 2009; Luo et al. 2012) in order to avoid the unfavorable numerical issues and improve computational efficiency in the standard LSMs (Dijk et al. 2013; Makhija and Maute 2014). In particular, the parametric level set method (PLSM) (Luo et al. 2007; Luo et al. 2008) has been demonstrated as one of the effective LSMs (Wang et al. 2014; Wang et al. 2015). The PLSM can not only retain the merits of most LSMs, but also overcome their numerical difficulties and enable the direct application of many gradient-based and more efficient optimization algorithms.

This paper will present a new topology optimization method using the Multi-Material Level Set (MM-LS) model (Wang et al. 2015) for design of multiphase multifunctional microstructures, in geometry with smoothed boundaries and distinct interfaces that may benefit fabrication of the topologically optimized microstructures. In the topological description model, the level set surfaces implicitly represent the structural boundaries as zero level sets. The material properties at any point in the domain are calculated according to a combination rule of different level set functions. This model provides a unique description for each phase and guarantees any point inside the domain only denoting one phase without overlaps. A number of $m$ level set functions are required to indicate $m+1$ distinct phases ( $m$ materials and 1 void phase).

In the topological shape optimization method to be developed, the numerical homogenization method will be applied to evaluate the effective properties of microstructures, while the PLSM is utilized to evolve shape and topology of the microstructure. It is known that the extreme thermos-mechanical property can be achieved by combining more than two phases with conventional materials, such as metals or plastics, into new types of composites. Typical numerical cases are used to demonstrate the effectiveness of the proposed method to design metamaterials with NPR and ZTE, as well as NPR and NTE (negative thermal expansion).

\section{Topological optimization method}

\subsection{Parameterization of multiple level set functions}

In LSMs, the design boundary is embedded implicitly into a higher-dimensional level set function $\Phi(\mathbf{x})$ with Lipschitz continuity as its zero level set (Osher and Sethian 1988; Sethian 1999) as follows:

$$
\left\{\begin{array}{lll}
\Phi^{I}(\mathbf{x})>0 & \forall \mathbf{x} \in \Omega^{I} \backslash \partial \Omega^{I} & \text { (Solid) } \\
\Phi^{I}(\mathbf{x})=0 & \forall \mathbf{x} \in \partial \Omega^{I} & \text { (Boundary) } \\
\Phi^{I}(\mathbf{x})<0 & \forall \mathbf{x} \in D \backslash\left(\Omega^{I} \cup \partial \Omega^{I}\right) & \text { (Void) }
\end{array}\right.
$$


where $D$ is the reference domain including all admissible shapes of the microstructure, $I=1, \ldots, m$ are the level set functions, and $\Phi^{I}(\mathbf{x})$ is $I^{\text {th }}$ the level set function. $\Omega^{I}$ is the domain having positive level set function values, $\partial \Omega^{I}$ denotes the design boundary of the $I^{\text {th }}$ level set function, and $m$ is the total number of the level set functions. Equation (1) defines the solid, boundary and void regions respectively by using the positive values, zero values and negative values, with a combined representation of different level set functions, rather than the representation of a single level set function.

Introducing pseudo-time $t$ to enable dynamic motion of the $\Phi(\mathbf{x}, t)$, and differentiating both sides of $\Phi(\mathbf{x}, t)$ with respect to $t$, will generate the Hamilton-Jacobi (H-J) equation (Sethian 1999; Osher and Fedkiw 2003)

$$
\frac{\partial \Phi^{I}(\mathbf{x}, t)}{\partial t}-\mathrm{v}_{\mathrm{n}}^{I}\left|\nabla \Phi^{I}(\mathbf{x}, t)\right|=0, \quad(I=1,2, \ldots, m)
$$

where $\mathrm{v}_{\mathrm{n}}^{I}$ is the normal velocity of the $I^{\text {th }}$ level set function. It is noted that only the normal component of the velocity contributes to the motion of the boundary. In the conventional level set methods, the details for getting numerical solution of the above H-J can be found in (Wang, Wang, and Guo 2003; Allaire, Jouve, and Toader 2004; Sethian 1999; Osher and Fedkiw 2003).

In the PLSM (Luo et al. 2007; Luo et al. 2008), the compactly supported radial basis function (CS-RBF) (Wendland 2006) has usually been applied to interpolate the level set surfaces. The CS-RBFs have drawn much attention in multivariate interpolations of scattered data, due to the positive definiteness and sparseness of the interpolation matrices under certain conditions, as well as the desired continuity of the interpolant. These favorable features make them attractive in the level set-based methods. It is noted that the application of different CS-RBFs, e.g. CS-RBFs with C2, C4 and C6 continuities (Wendland 2006), to the level-set based topology optimization problems have previously been investigated in detail(Luo et al. 2007; Luo et al. 2008). The results show that at least the Wendland C2, C4 and C6 can give similar topological designs, although the sensitivity with respect to the compact support radius will increase along with the increase in the differentiability order. The CS-RBFs with C2 smoothness are used in this paper.

The original level set function can be described by the interpolation of the CS-RBFs at their pre-specified knots over the whole design domain, as follows:

$$
\boldsymbol{\Phi}^{I}(\mathbf{x}, t)=\boldsymbol{\varphi}(\mathbf{x}) \boldsymbol{c}^{I}(t)=\sum_{i=1}^{N} \varphi_{i}(\mathbf{x}) c_{i}^{I}(t)(I=1, \ldots, m)
$$

with the vector of the shape functions defined as follows:

$$
\boldsymbol{\varphi}(\mathbf{x})=\left[\varphi_{1}(\mathbf{x}), \varphi_{2}(\mathbf{x}), \ldots, \varphi_{N}(\mathbf{x})\right] \in \mathfrak{R}^{N}
$$

and the expansion coefficient vector is given by 


$$
\boldsymbol{c}^{I}(t)=\left[c_{1}^{I}(t), c_{2}^{I}(t), \ldots, c_{N}^{I}(t)\right]^{\mathrm{T}} \in \mathfrak{R}^{N}
$$

where $N$ is the total number of the CS-RBF knots in the design domain. $\boldsymbol{\varphi}_{i}(\mathbf{x})$ is the $i^{\text {th }}$ CS-RBF $(i=1, \ldots \mathrm{N})$.

Since the CS-RBF shape functions are spatial only, while their corresponding expansion coefficients are time dependent, the interpolation of the level set function by the CS-RBFs leads to a separation of space and time. Here the same set of CS-RBFs is employed to interpolate each implicit level set function. Thus, the decoupling of the time and space terms in the H-J PDE will result in

$$
\boldsymbol{\varphi}(\mathbf{x}) \frac{\mathrm{d} c^{I}(t)}{\mathrm{d} t}-\mathbf{v}_{\mathrm{n}}^{I}\left|\nabla \varphi(\mathbf{x}) c^{I}(t)\right|=0
$$

The normal velocity $\mathbf{v}_{\mathrm{n}}^{I}$ is related to the time derivative of the expansion coefficients, given as follows:

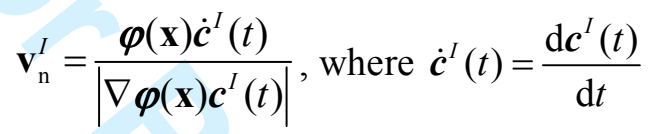

From the above velocity field after the interpolation, it can be seen that all the terms involved in the velocity vector are actually evaluated over the whole domain, which will lead to a natural extension of the normal velocity field from the boundary to the whole domain.

The original level set equation is now parameterized into a system of algebraic equations. The only unknowns are the expansion coefficients of the CS-RBF interpolant, defined as the design variables, to be updated using appropriate optimization algorithms. Hence, the propagation of the boundaries at the zero level sets just requires iteratively updating the interpolant expansion coefficients.

\subsection{Multi-phase level set model of materials}

The multi-material level set model of structures can be extended to the design of metamaterials (Wang et al. 2015). Each material phase is represented by a combination of different level set functions. For instance, for a composite with one, two or three solid phases, the elastic stiffness $\boldsymbol{C}(\mathbf{x})$ at any point $\mathbf{x}$ is given by

$$
\begin{aligned}
& \boldsymbol{C}^{(1)}(\mathbf{x}, \Phi)=H\left(\Phi^{1}\right) \boldsymbol{C}^{1} \\
& \boldsymbol{C}^{(2)}(\mathbf{x}, \Phi)=H\left(\Phi^{1}\right)\left(1-H\left(\Phi^{2}\right)\right) \boldsymbol{C}^{1}+H\left(\Phi^{1}\right) H\left(\Phi^{2}\right) \boldsymbol{C}^{2} \\
& \boldsymbol{C}^{(3)}(\mathbf{x}, \Phi)=H\left(\Phi^{1}\right)\left(1-H\left(\Phi^{2}\right)\right) \boldsymbol{C}^{1}+H\left(\Phi^{1}\right) H\left(\Phi^{2}\right)\left(1-H\left(\Phi^{3}\right)\right) \boldsymbol{C}^{2}+H\left(\Phi^{1}\right) H\left(\Phi^{2}\right) H\left(\Phi^{3}\right) \boldsymbol{C}^{3}
\end{aligned}
$$

where $H\left(\Phi^{I}\right)$ is the Heaviside function corresponding to the $m^{\text {th }}$ level set function. For a case with $m$ solid phase materials, the elastic stiffness $\boldsymbol{C}(\mathbf{x})$ can be obtained in a similar way by following the above model. This definition is in line with the level set representation model given in Equation (1). 
Since the thermal expansion coefficient $\alpha(\mathbf{x})$ is only related to the mixture of materials, it will have the form

$$
\begin{aligned}
& \alpha^{(1)}(\mathbf{x}, \Phi)=\alpha^{1}, \\
& \alpha^{(2)}(\mathbf{x}, \Phi)=\left(1-H\left(\Phi^{2}\right)\right) \alpha^{1}+H\left(\Phi^{2}\right) \alpha^{2}, \\
& \alpha^{(3)}(\mathbf{x}, \Phi)=\left(1-H\left(\Phi^{2}\right)\right) \alpha^{1}+H\left(\Phi^{2}\right)\left(1-H\left(\Phi^{3}\right)\right) \alpha^{2}+H\left(\Phi^{2}\right) H\left(\Phi^{3}\right) \alpha^{3},
\end{aligned}
$$

As illustrated by the above MM-LS model, a number of $m+1$ material phases can be represented by using a number of $m$ level set functions. The distribution of multiple materials can be described in the design domain. As given in Figure 1, the level set function $\Phi^{1}$ is firstly used to distinguish the solid (blue, red and green regions $\Phi^{1}>0$ ) and the void phase (grey $\Phi^{1}<0$ ), $\Phi^{2}$ is then applied to determine the first material phase (blue region $\Phi^{1}>0$ and $\Phi^{2}<0$ ) from the material phases, and $\Phi^{3}$ is finally used to identify the second material phase (red region $\Phi^{1}>0, \Phi^{2}>0$ and $\Phi^{3}<0$ ) and the third material phase (green region $\Phi^{1}>0, \Phi^{2}>0$ and $\Phi^{3}>0$ ) .

It is noted that the MM-LS is actually a general model, which is applicable to more than 3 material phases. In this study, for simplicity but without loss of generality, we have only selected three phases. For the case with $\mathrm{k}(>=3)$ materials, the elastic stiffness $\mathrm{C}^{(\mathrm{k})}(\mathrm{x})$ and the thermal expansion coefficient $\alpha^{(\mathrm{k})}(\mathrm{x})$ can be obtained according to the similar rules given by Eqs. (8) and (9).

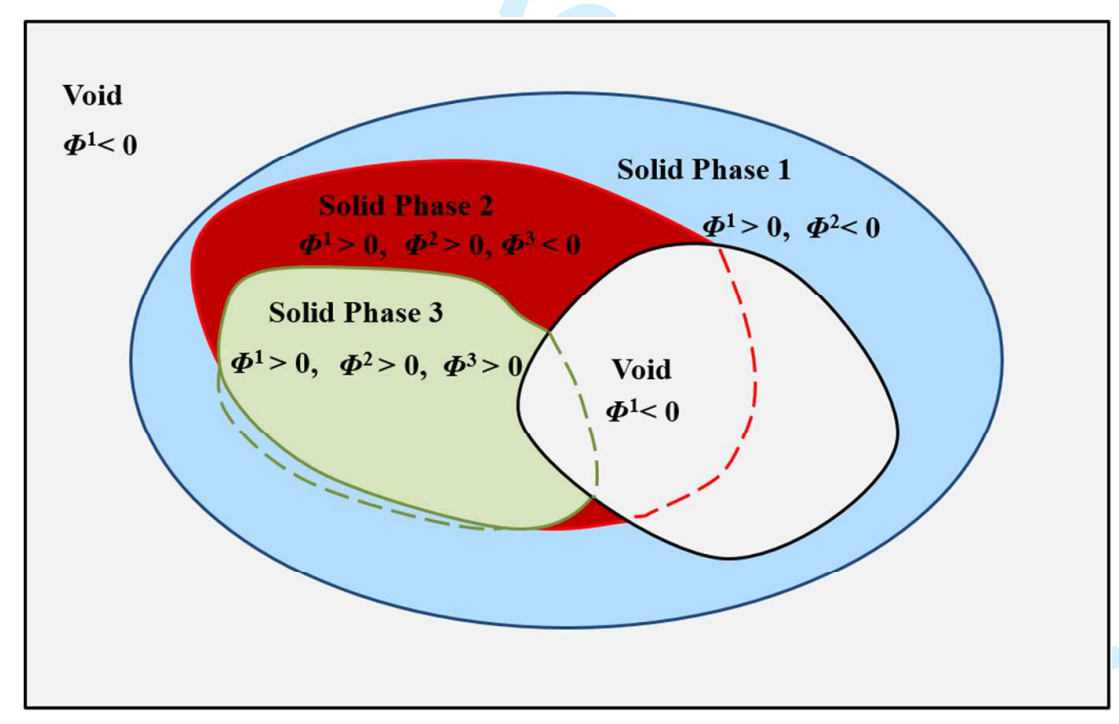

Figure 1: Representation of a multi-material design domain

For the problem studied in this paper, the elasticity constant $C$ and thermal strain coefficient $\alpha$ at any computational point $\mathbf{x}$ inside the domain can be written as a function of the values of the $\Phi^{1}$ and $\Phi^{2}$ :

$$
C_{i j k l}(\mathbf{x}, \Phi)=H\left(\Phi^{1}\right)\left(1-H\left(\Phi^{2}\right)\right) C_{i j k l}^{1}+H\left(\Phi^{1}\right) H\left(\Phi^{2}\right) C_{i j k l}^{2}
$$




$$
\alpha_{i j}(\mathbf{x}, \Phi)=\left(1-H\left(\Phi^{2}\right)\right) \alpha_{i j}^{1}+H\left(\Phi^{2}\right) \alpha_{i j}^{2}
$$

where $\mathrm{H}$ is a smoothed Heaviside function, normally given as follows:

$$
H(\Phi(\mathrm{x}))=\left\{\begin{array}{cc}
\theta & \mathrm{x}<-\Delta \\
\frac{3(1-\theta)}{4}\left(\frac{\Phi(\mathrm{x})}{\Delta}-\frac{\Phi(\mathrm{x})^{3}}{3 \Delta^{3}}\right)+\frac{1+\theta}{2} & -\Delta \leq \mathrm{x}<\Delta \\
1 & \mathrm{x} \geq \Delta
\end{array}\right.
$$

where $\theta$ is a small positive number to avoid numerical singularity, and $\Delta$ is the width for the Heaviside function. Here, the Heaviside function is smoothed to facilitate the calculation of the first-order derivatives of the objective function.

It should be noted that the smoothed level set function will naturally smear the exact boundaries of level sets, and may impact the accurate representation of the original shape and distinct interface. So the validity of the constitutive equation may not exactly ensure accurate capture of the Hashin-Shtrikman bounds (Bensøe and Sigmund 2003). There have been some methods developed to overcome this issue, e.g. the multiphase piecewise constant level set method (Luo et al. 2009; Wei and Wang 2009). The MM-LS model employs continuous level set functions to describe each phase given in Eq. (8), and thus the advantages of the level set method can be retained. Hence, the MM-LS is different from the mixture model in the material density distribution methods, e.g. (Sigmund and Torquato 1997; Bensøe and Sigmund 1999).

\subsection{Topological shape optimization of metamaterials}

The topological optimization will be performed in a unique cell $Y$ defined as the design domain. The effective elastic and thermoelastic properties can be evaluated by using the homogenization method as:

$$
\begin{gathered}
C_{i j k l}^{\mathrm{H}}=\frac{1}{|Y|} \int_{Y}\left(\varepsilon_{p q}^{0(i j)}-\varepsilon_{p q}^{*}\left(\chi^{i j}\right)\right) C_{p q r s}\left(\varepsilon_{r s}^{0(k l)}-\varepsilon_{r s}^{*}\left(\chi^{k l}\right)\right) \mathrm{d} Y \\
\beta_{i j}^{\mathrm{H}}=\frac{1}{|Y|} \int_{Y}\left(\alpha_{p q}-\varepsilon_{p q}^{*}(\Theta)\right) C_{p q k l}\left(\varepsilon_{k l}^{0(i j)}-\varepsilon_{k l}^{*}\left(\chi^{i j}\right)\right) \mathrm{d} Y \\
\alpha_{i j}^{\mathrm{H}}=\left(C_{i j k l}^{\mathrm{H}}\right)^{-1} \beta_{k l}^{\mathrm{H}}
\end{gathered}
$$

where $C_{i j k l}^{\mathrm{H}}$ is the effective elasticity tensor, $\beta_{i j}^{\mathrm{H}}$ is the effective thermal stress tensor, $\alpha_{i j}^{\mathrm{H}}$ is the effective thermal strain tensor, $C_{p q r s}$ is the locally varying elasticity tensor, $\varepsilon^{0}$ is the unit test strain field ( 3 for $2 \mathrm{D}$ and 6 for 3D), $|Y|$ is the volume of the cell, $\alpha_{p q}$ is the locally varying thermal strain tensor corresponding to a unit strain caused by a unit thermal load, $\varepsilon^{*}\left(\chi^{k l}\right)$ denote locally vary strain fields, and $\varepsilon^{*}(\Theta)$ are the strain field based on the displacement field for a unit thermal load. The displacement fields $\chi^{k l}$ and $\Theta$ can be obtained by solving the following equations:

$$
\int_{Y}\left(\varepsilon_{p q}^{0(k l)}-\varepsilon_{p q}^{*}\left(\chi^{k l}\right)\right) C_{p q r s} \varepsilon_{r s}(\tau) \mathrm{d} Y=0, \forall \tau \in \bar{U}(Y)
$$




$$
\int_{Y}\left(\alpha_{p q}-\varepsilon_{p q}^{*}(\Theta)\right) C_{p q r s} \varepsilon_{r s}(\tau) \mathrm{d} Y=0, \forall \tau \in \bar{U}(Y)
$$

where $\tau$ is the virtual displacement field.

This work aims to optimize the shape and topology of the microstructure to achieve the expected effective properties for both the thermal strain tensor $\alpha_{i j}^{o b j}$ and the elasticity tensor $C_{i j k l}^{o b j}$ under the constraint of a given amount of materials in the design domain (3-phase). The symmetries, such as orthotropy, square symmetry or isotropy of the resulting materials, will also be considered in the optimization.

The optimization problem with multiple materials is then formulated as:

$$
\begin{cases}\text { Find : } c_{i}^{1}, c_{i}^{2}(i=1,2, \ldots, N) \\ \text { Minimize: } f=\frac{1}{2} \sum_{i, j=1}^{d} \omega_{i j}\left(\alpha_{i j}^{\mathrm{H}}-\alpha_{i j}^{O b j}\right)^{2}+\frac{1}{2} \sum_{i, j, k, l=1}^{d} \eta_{i j k l}\left(C_{i j k l}^{\mathrm{H}}-C_{i j k l}^{O b j}\right)^{2} \\ \text { Subject to: } \\ \qquad(\boldsymbol{u}, \boldsymbol{v}, \varphi)=l(\boldsymbol{v}, \varphi), \quad \forall \boldsymbol{v} \in U_{\mathrm{ad}} \\ V_{\min }^{1} \leq V^{1} \leq V_{\max }^{1} \\ V_{\min }^{2} \leq V^{2} \leq V_{\max }^{2} \\ c_{\min }^{1} \leq c_{i}^{1} \leq c_{\max }^{1} \\ c_{\min }^{2} \leq c_{i}^{2} \leq c_{\max }^{2}\end{cases}
$$

where $\omega_{i j}$ and $\eta_{i j k l}$ are the weighting factors associated with the corresponding thermal strain tensors and the elasticity tensor; $\boldsymbol{u}$ is the displacement field; $\boldsymbol{v}$ is the virtual displacement and $U_{\text {ad }}$ is the set of kinematically admissible displacements; $V_{\min }^{1}, V_{\max }^{1}, V_{\min }^{2}$ and $V_{\max }^{2}$ are lower and upper bounds to limit the volume fractions of solid phase 1 and solid phase 2 respectively; $c_{\min }^{1}, c_{\max }^{1}, c_{\min }^{2}$ and $c_{\max }^{2}$ are the lower and upper bounds of the two sets of design variables.

In this problem, the number of constraints is equal to the number of solid phases. The constraints impose a restriction on the overall usage of material, as well as the usage of each individual solid material, to achieve better convergence. In this case, the volume fraction of two solid phases can be expressed as

$$
V^{1}=\frac{1}{|Y|} \int_{Y} H\left(\Phi^{1}\right) \mathrm{d} Y \text { and } V^{2}=\frac{1}{|Y|} \int_{Y} H\left(\Phi^{1}\right) H\left(\Phi^{2}\right) \mathrm{d} Y
$$

where the first constraint refers to the overall usage of solid phases 1 and 2, while the second, which is a nonlinear constraint, restricts the usage of the solid phase 2. The order of nonlinearity will increase when more solid phases are included which may cause oscillations of the constraint. In this case, additional 
numerical techniques may be required to achieve a stable convergence. In addition, as aforementioned, the Heaviside function used here is smoothed, and thus the volume fractions calculated are approximate.

Due to the interpolation of the level set function, only the expansion coefficients of the interpolant are required to be optimized using appropriate optimization algorithms such as the Method of Moving Asymptotes (MMA) (Svanberg 1987). The implementation of MMA requires the first-order derivatives of the objective function and constraints with respect to the design variables. Considering variation of the moving boundary due to the pseudo-time $t$, the sensitivity can be computed by the shape derivative analysis method and the adjoint method (Choi and Kim 2005).

\subsection{Shape derivative analysis}

The first-order derivative of the objective function with respect to the design variable $c^{I}$ is obtained as

$$
\frac{d f}{d \boldsymbol{c}^{I}}=\sum_{i, j=1}^{d} \omega_{i j}\left(\alpha_{i j}^{\mathrm{H}}-\alpha_{i j}^{O b j}\right) \frac{\partial \alpha_{i j}^{\mathrm{H}}}{\partial \boldsymbol{c}^{I}}+\sum_{i, j, k, l=1}^{d} \eta_{i j k l}\left(C_{i j k l}^{\mathrm{H}}-C_{i j k l}^{O b j}\right) \frac{\partial C_{i j k l}^{\mathrm{H}}}{\partial \boldsymbol{c}^{I}}
$$

In the above, in order to obtain both $\partial C_{i j k l}^{\mathrm{H}} / \partial c^{I}$ and $\partial \alpha_{i j}^{\mathrm{H}} / \partial c^{I}$, we need to first calculate the derivative of $C_{i j k l}^{\mathrm{H}}$ and $\beta_{i j}^{\mathrm{H}}$ with respect to the pseudo time $t$.

Let $J(\Omega)$ be a shape function of the domain $\Omega$ (Choi and Kim 2005), whose material derivative along a given direction can be defined, as follows

$$
\frac{d J}{d t}=\lim _{t \rightarrow 0} \frac{1}{t}\left[J\left(\Omega_{t}\right)-J(\Omega)\right]
$$

The material derivatives of $C_{i j k l}^{\mathrm{H}}$ are (Allaire, Jouve, and Toader 2004) and (Choi and Kim 2005):

$$
\begin{aligned}
\frac{d C_{i j k l}^{\mathrm{H}}}{d t}= & \frac{1}{|Y|} \int_{Y}\left(\varepsilon_{p q}^{0}-\varepsilon_{p q}^{*}\left(\chi^{i j}\right)\right) \frac{\partial C_{p q r s}}{\partial \Phi^{I}}\left(\varepsilon_{r s}^{0}-\varepsilon_{r s}^{*}\left(\chi^{k l}\right)\right) v_{\mathrm{n}}^{I} \mathrm{~d} Y-\frac{2}{|Y|} \int_{Y}\left(\varepsilon_{p q}^{0}-\varepsilon_{p q}^{*}\left(\chi^{i j}\right)\right) C_{p q r s}\left(\varepsilon_{r s}^{*}\left(\delta \chi^{k l}\right)\right) v_{\mathrm{n}}^{I} \mathrm{~d} Y \\
\frac{d \beta_{i j}^{\mathrm{H}}}{d t}= & \frac{1}{|Y|} \int_{Y}\left(\alpha_{p q}-\varepsilon_{p q}^{*}(\Theta)\right) \frac{\partial C_{p q r s}}{\partial \Phi^{I}}\left(\varepsilon_{r s}^{0(i j)}-\varepsilon_{r s}^{*}\left(\chi^{i j}\right)\right) v_{\mathrm{n}}^{I} \mathrm{~d} Y-\frac{1}{|Y|} \int_{Y}\left(\alpha_{p q}-\varepsilon_{p q}^{*}(\Theta)\right) C_{p q r s} \varepsilon_{r s}^{*}\left(\delta \chi^{i j}\right) v_{\mathrm{n}}^{I} \mathrm{~d} Y \\
& -\frac{1}{|Y|} \int_{Y} \varepsilon_{p q}^{*}(\delta \Theta) C_{p q r s}\left(\varepsilon_{r s}^{0(i j)}-\varepsilon_{r s}^{*}\left(\chi^{i j}\right)\right) v_{\mathrm{n}}^{I} \mathrm{~d} Y
\end{aligned}
$$

where $v_{n}$ is a velocity vector, denoting the perturbation direction of the domain boundaries, is the normal velocity of the $I^{\text {th }}$ material boundary, which can be obtained by Eq. (7); $\partial C_{p q r s} / \partial \Phi^{I}$ can be calculated from Eqs. (8) and (9). In the above equations, $\delta \chi^{k l}$ and $\delta \Theta$ are the derivatives of $\chi^{k l}$ and $\Theta$ with respect to $\Phi^{I}$. Based on the derivative given in Eqs. (16) and (17) with respect to $\Phi^{I}$, we can obtain 


$$
\begin{gathered}
\frac{1}{|Y|} \int_{Y} \varepsilon_{p q}^{*}(\tau) C_{p q r s}\left(\varepsilon_{r s}^{*}\left(\delta \chi^{k l}\right)\right) v_{\mathrm{n}}^{I} \mathrm{~d} Y=\frac{1}{|Y|} \int_{Y} \varepsilon_{p q}^{*}(\tau) \frac{\partial C_{p q r s}}{\partial \Phi^{I}}\left(\varepsilon_{r s}^{0}-\varepsilon_{r s}^{*}\left(\chi^{k l}\right)\right) v_{\mathrm{n}}^{I} \mathrm{~d} Y \\
\frac{1}{|Y|} \int_{Y} \varepsilon_{p q}^{*}(\delta \Theta) C_{p q r s} \varepsilon_{r s}^{*}(\tau) v_{\mathrm{n}}^{I} \mathrm{~d} Y=\frac{1}{|Y|} \int_{Y}\left(\varepsilon_{p q}-\varepsilon_{p q}^{*}(\Theta)\right) \frac{\partial C_{p q r s}}{\partial \Phi^{I}} \varepsilon_{r s}^{*}(\tau) v_{\mathrm{n}}^{I} \mathrm{~d} Y
\end{gathered}
$$

Substituting Eqs. (24) and (25) into Eqs. (22) and (23) with an arbitrary $t$, we have

$$
\begin{aligned}
& \frac{d C_{i j k l}^{\mathrm{H}}}{d t}=-\frac{1}{|Y|} \int_{Y}\left(\varepsilon_{p q}^{0}-\varepsilon_{p q}^{*}\left(\chi^{i j}\right)\right) \frac{\partial C_{p q r s}}{\partial \Phi^{I}}\left(\varepsilon_{r s}^{0}-\varepsilon_{r s}^{*}\left(\chi^{k l}\right)\right) v_{\mathrm{n}}^{I} \mathrm{~d} Y \\
& \frac{d \beta_{i j}^{\mathrm{H}}}{d t}=-\frac{1}{|Y|} \int_{Y}\left(\alpha_{p q}-\varepsilon_{p q}^{*}(\Theta)\right) \frac{\partial C_{p q r s}}{\partial \Phi^{I}}\left(\varepsilon_{r s}^{0(i j)}-\varepsilon_{r s}^{*}\left(\chi^{i j}\right)\right) v_{\mathrm{n}}^{I} \mathrm{~d} Y
\end{aligned}
$$

Then, subjecting the previously defined $v_{\mathrm{n}}^{I}$ into the above Eqs. (26) and (27) will lead to

$$
\begin{aligned}
& \frac{d C_{i j k l}^{\mathrm{H}}}{d t}=-\frac{1}{|Y|} \int_{Y}\left(\varepsilon_{p q}^{0}-\varepsilon_{p q}^{*}\left(\chi^{i j}\right)\right) \frac{\partial C_{p q r s}}{\partial \Phi^{I}}\left(\varepsilon_{r s}^{0}-\varepsilon_{r s}^{*}\left(\chi^{k l}\right)\right) \frac{\varphi(\mathbf{x})}{\left|\nabla \Phi^{I}\right|} \mathrm{d} Y \frac{\mathrm{d} \boldsymbol{c}^{I}(t)}{\mathrm{d} t} \\
& \frac{d \beta_{i j}^{\mathrm{H}}}{d t}=-\frac{1}{|Y|} \int_{Y}\left(\alpha_{p q}-\varepsilon_{p q}^{*}(\Theta)\right) \frac{\partial C_{p q r s}}{\partial \Phi^{I}}\left(\varepsilon_{r s}^{0(i j)}-\varepsilon_{r s}^{*}\left(\chi^{i j}\right)\right) \frac{\varphi(\mathbf{x})}{\left|\nabla \Phi^{I}\right|} \mathrm{d} Y \frac{\mathrm{d} \boldsymbol{c}^{I}(t)}{\mathrm{d} t}
\end{aligned}
$$

To eliminate $\mathrm{d} c^{I}(t) / \mathrm{d} t$, the derivative of $C_{i j k l}^{\mathrm{H}}$ and $\beta_{i j}^{\mathrm{H}}$ with respect to $t$ can also be given via the chain-rule:

$$
\frac{d C_{i j k l}^{\mathrm{H}}}{d t}=\frac{\partial C_{i j k l}^{\mathrm{H}}}{\partial c^{I}} \frac{\partial \boldsymbol{c}^{I}}{\partial t} ; \frac{d \beta_{i j}^{\mathrm{H}}}{d t}=\frac{\partial \beta_{i j}^{\mathrm{H}}}{\partial c^{I}} \frac{\partial \boldsymbol{c}^{I}}{\partial t}
$$

By comparing the corresponding terms in Eq. (30) with that given in Eqs. (28) and (29) yields

$$
\begin{aligned}
\frac{\partial C_{i j k l}^{\mathrm{H}}}{\partial \boldsymbol{c}^{I}} & =-\frac{1}{|Y|} \int_{Y}\left(\varepsilon_{p q}^{0}-\varepsilon_{p q}^{*}\left(\chi^{i j}\right)\right) \frac{\partial C_{p q r s}}{\partial \Phi^{I}}\left(\varepsilon_{r s}^{0}-\varepsilon_{r s}^{*}\left(\chi^{k l}\right)\right) \frac{\boldsymbol{\varphi}(\mathbf{x})}{\left|\nabla \Phi^{I}\right|} \mathrm{d} Y \\
\frac{\partial \beta_{i j}^{\mathrm{H}}}{\partial \boldsymbol{c}^{I}} & =-\frac{1}{|Y|} \int_{Y}\left(\alpha_{p q}-\varepsilon_{p q}^{C^{*}}\right) \frac{\partial C_{p q r s}}{\partial \Phi^{I}}\left(\varepsilon_{r s}^{0(i j)}-\varepsilon_{r s}^{*}\left(\chi^{i j}\right)\right) \frac{\boldsymbol{\varphi ( \mathbf { x } )}}{\left|\nabla \Phi^{I}\right|} \mathrm{d} Y
\end{aligned}
$$

and recalling Eq. (15), we will have

$$
\frac{\partial \alpha_{i j}^{\mathrm{H}}}{\partial c^{I}}=\frac{\partial\left(C_{i j k l}^{\mathrm{H}}\right)^{-1}}{\partial c^{I}} \beta_{k l}^{\mathrm{H}}+\left(C_{i j k l}^{\mathrm{H}}\right)^{-1} \frac{\partial \beta_{k l}^{\mathrm{H}}}{\partial c^{I}}
$$

Similarly, the derivative of the volume constraints can also be obtained, as follows:

$$
\frac{\partial V^{1}}{\partial \boldsymbol{c}^{1}}=\frac{1}{|Y|} \int_{Y} \delta\left(\Phi^{1}\right) \varphi(\mathbf{x}) \mathrm{d} Y ; \quad \frac{\partial V^{2}}{\partial c^{1}}=\frac{1}{|Y|} \int_{Y} \delta\left(\Phi^{1}\right) H\left(\Phi^{2}\right) \varphi(\mathbf{x}) \mathrm{d} Y ; \quad \frac{\partial V^{2}}{\partial \boldsymbol{c}^{2}}=\frac{1}{|Y|} \int_{Y} H\left(\Phi^{1}\right) \delta\left(\Phi^{2}\right) \varphi(\mathbf{x}) \mathrm{d} Y
$$

where the Dirac delta function $\delta(\Phi)=\partial H(\Phi) / \partial \Phi$. 
In Equation (34), the use of the Heaviside function will result in the use of its first-order derivatives, namely the Dirac delta function. Hence, the sensitivities will be infinity at the level set boundary while zero elsewhere. Since a smoothed Heaviside function has been used in the numerical implementation, it is still reasonable to use the delta function in the sensitivity analysis to enable the decrease of the objective.

\section{Numerical Examples}

In the following examples, an 'ersatz material' model (Allaire, Jouve and Toader 2004) is used to approximate strains and material properties for those elements crossed by the moving level set boundary. A cell with periodic boundary conditions is regarded as the design domain. Geometric symmetries of the cell are also considered in order to achieve orthotropic or balanced orthotropic materials. The metamaterials designed in this paper are subject to the plane stress condition, and the geometric symmetries of the cell are limited to achieve plane orthotropic materials with cubic symmetry. For simplicity, the level set knots are assumed to be identical with the element nodes, resulting in $61 \times 61$ total design variables. The convergence criterion is that the difference of two successive objective function values is less than 0.0001 , or the maximum iteration number is no more than 200 for the numerical cases in this paper. The radius of CS-RBF is set to 1.5 times of the average distance between CS-RBF knots because an appropriate radius of compact support is essential for an efficient trade-off between interpolation and computational efficiency. To obtain materials with extreme Poisson's ratio and thermal expansion coefficient, different weighting factors will be used for different design requirements of the NPR and ZTE. By changing the weighting factors, we can obtain final material designs with different values of NPR and ZTE.

\section{Numerical Example 1}

In Case (1) we design an orthotropic material to achieve a NPR of -0.5 and ZTE. A total of two level set functions are used to denote the multi-material design domain. Note that the first level set function represents the overall solid phases rather than any single material phase. The two artificial solid materials are assumed to have Young's moduli: $E^{1}=E^{2}=1$, Poisson's ratios: $v^{1}=v^{2}=0.3$, and the thermal expansion coefficients $\alpha^{1}=1$ and $\alpha^{2}=10$. Horizontal and vertical geometric symmetries are included, the total material usage $V^{1}=0.35$ and the material usage for solid phase 2 is in the range $0.15>=V^{2}>=0.1$.

The initial parameters such as the weighting factors, expected objective values and effective properties to be achieved by the optimization are given in Table 1. The designs and the convergence are shown in Figs 2 and 3, the level set contours are given in Fig. 4, and the two solid phases are shown in Fig. 5. It is noted that the different values given by Fig. 5 represent $\Phi^{1(\text { or } 2)}>0, \Phi^{1(\text { or } 2)}=0$ and $\Phi^{1(\text { or } 2)}<0$, respectively. In Fig. 5 , the materials corresponding to the red regions labeled Solid 1denote the solid phase $1\left(\Phi^{1}>0 \& \Phi^{2}<0\right)$, while the 
material in the blue regions labeled Solid 2 represent the solid phase $2\left(\Phi^{1}>0 \& \Phi^{2}>0\right)$. The above observations are exactly in line with the definition of multi-materials in the design domain (Fig.1). For instance, for three material phases, both $\Phi^{1}>0$ and $\Phi^{2}>0$ will determine the solid 2 .

In this example it seems that the optimized design is topologically equivalent to the initial design with 9 holes, and the design appears to have only experienced shape changes. However, upon close observation of the intermediate designs shown in Figure 2, we find that new holes are indeed created and merged during the shape and topology optimization process. Hence, both the shape and topology of the initial microstructure have in fact experienced changes during the optimization. Based on the results, it can be seen that the topology of the optimized design is a kind of porous microstructure with re-entrant geometries. It is known that the effective elastic and thermal properties of the microstructure depend on both the internal structural layout of the cell and the manner of deformation to enable rotating effects when loaded. It has been demonstrated that the re-entrant features are essential for NPR materials (Evans and Alderson 2000). Sigmund and Torquato $(1996,1997)$ also showed that extreme thermoelastic properties can be obtained from composites of three material phases ( 2 solids and 1 void), where one of the two solids responds more to thermal load than the other.

To demonstrate the deformation of the optimized structure, the displacements of the field nodes of a 3 by 3 array of the optimized base cell are plotted in Fig. 5. In Fig. 5(a), the 3 by 3 array is subjected to a horizontal force uniformly distributed along the right edge, while the left edge is constrained in the horizontal direction and the bottom edge is constrained in the vertical direction as a symmetrical displacement boundary. The negative Poisson's ratio effect can be seen in the structure with positive displacements in both horizontal and vertical directions (expanding). Fig. 5(b) shows the temperature change (thermal load) within the whole area, but almost no deformation can be seen in the plot due to the zero effective thermal expansion.

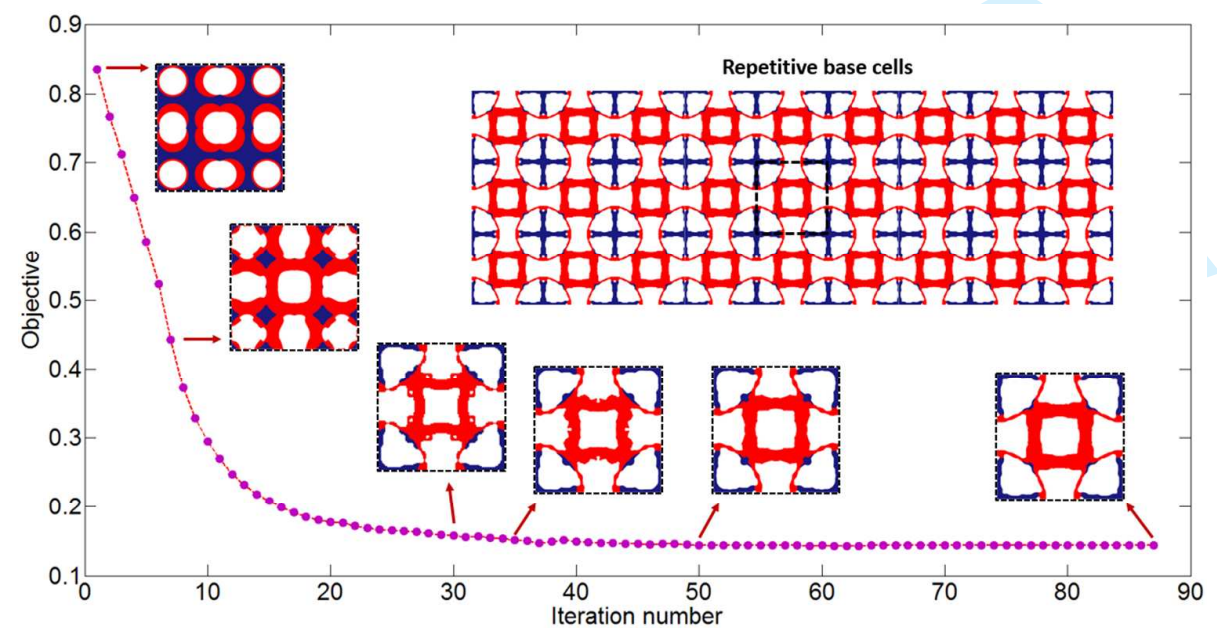

Figure 2: Convergence histories of the objective function 


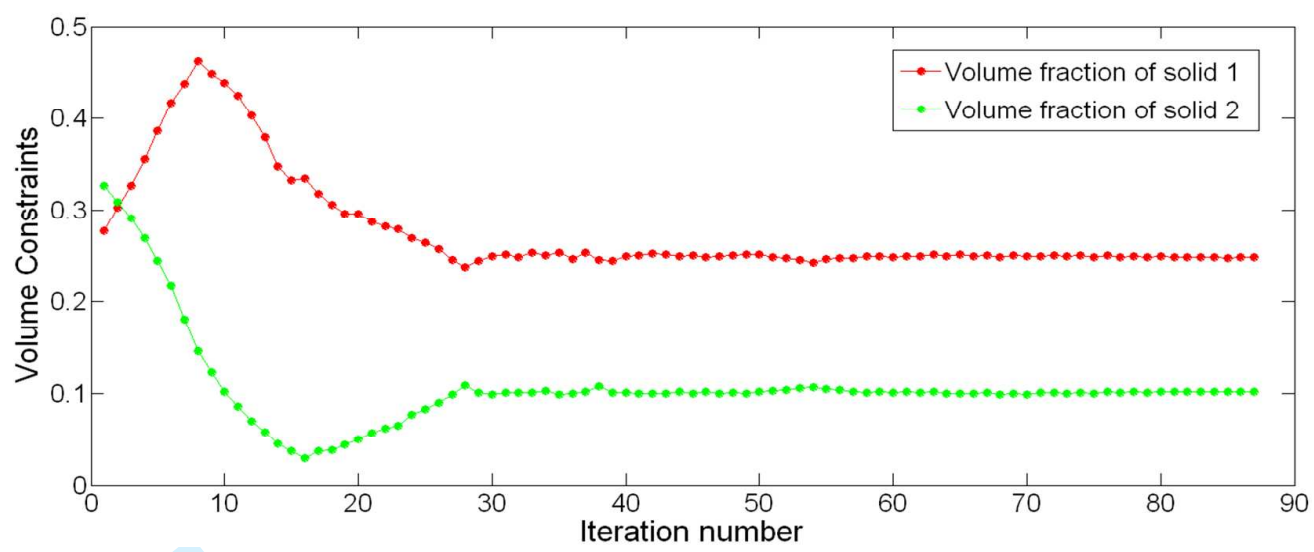

Figure 3. Convergence of two volume constraints

Table 1: Initial parameters and optimized results for Case (1)

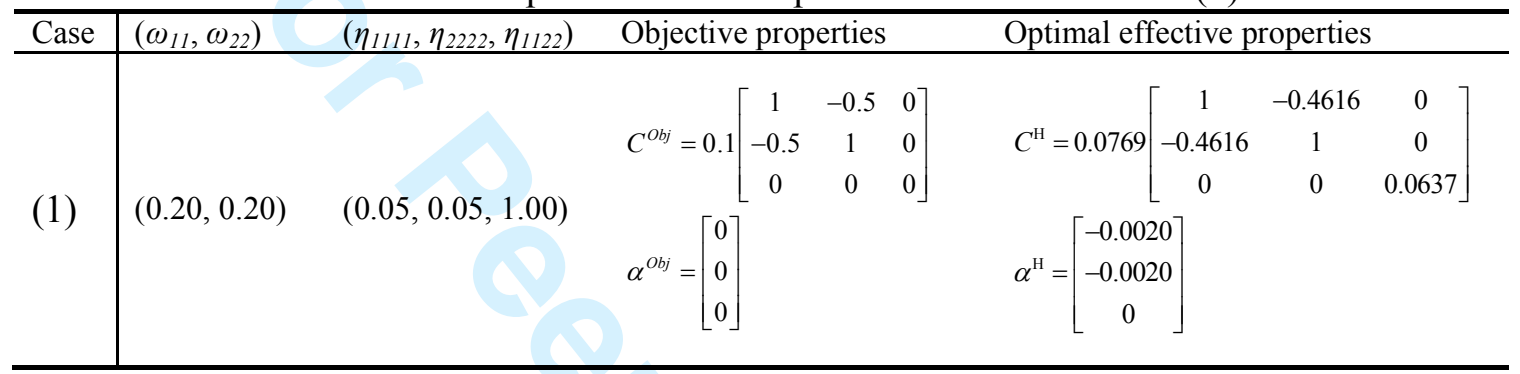

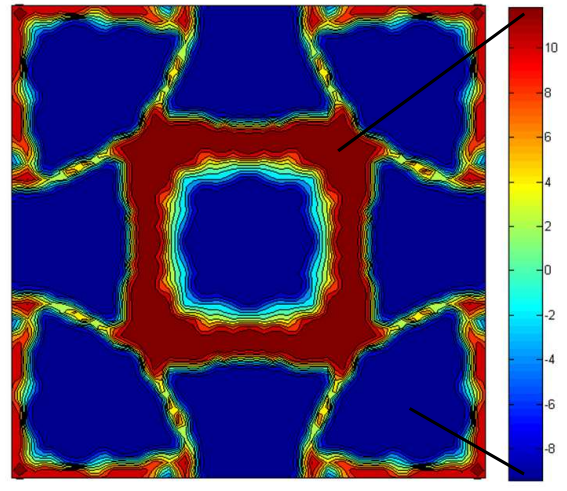

Figure 4. (a) Contours of LSF1 $\left(\Phi^{1}\right)$

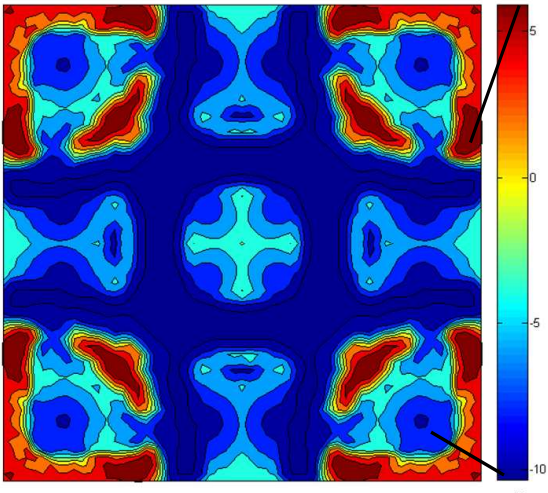

(b) Contours of LSF2 $\left(\Phi^{2}\right)$

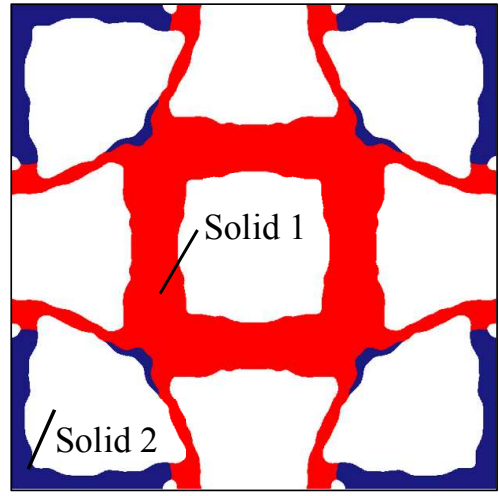

(c) Distribution of two solids:

Solid 1 ( $\Phi_{1}>0 \& \Phi_{2}<0$, Red color online) and Solid $2\left(\Phi_{1}>0 \& \Phi_{2}>0\right.$, Blue color online)

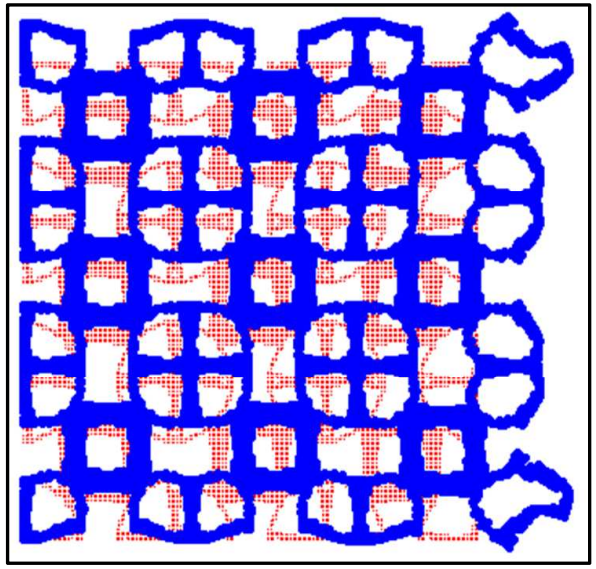

(a) Negative Poisson's ratio

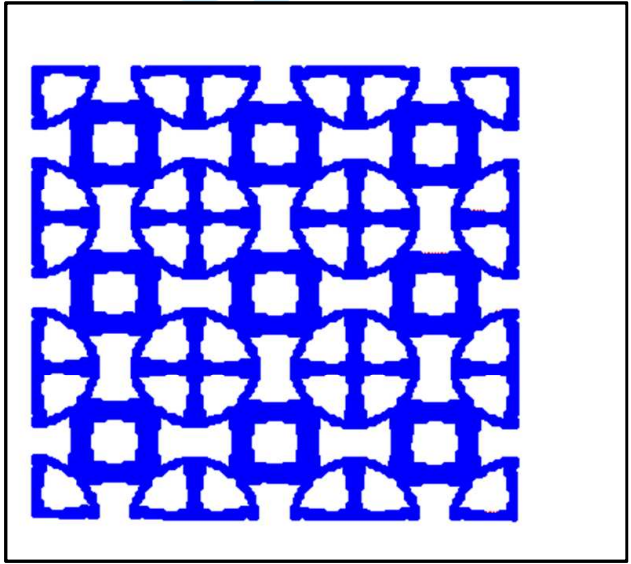

(b) Zero thermal expansion

Figure 5. Displacement plots of field nodes 


\section{Numerical Example 2}

The objective function of Case (2) is to design a balanced orthotropic (square symmetrical) metamaterial to have negative Poisson's ratio value -0.5 and zero thermal strain in both directions. The properties for two solid material phases and volume constraints are the same as that given in Case (1), but the initial designs are different. Other parameters are listed in Table 2. The convergence for the objective function and two constraints are given in Figs 6 and 7, the contours of two level set functions and the optimized design for solid phases are plotted in Fig. 8.

From the optimal effective properties in Tables 1 and 2, it can be seen that the Poisson's ratio and the thermal strain of the optimized design cannot exactly approach the expected property values. One of the possible reasons is that the effect of re-entrant structures is a key to enabling large rotating effects (rigidbody rotations) for a loaded microstructure (Grima et al. 2012). However, the topological design is actually a structural optimization method for continuum structures, which can only make use of structural elastic deformation (strain energy) to mimic rather than implement the rigid-body rotations. Hence, it is difficult to allow the microstructure to generate the same rotating deformation effect as rigid-link mechanisms. This may explain the phenomenon that during the optimization process point-to-point hinges or structural disconnections may occur at some local positions of the re-entrant structure. From the optimization, it can be found that the integrated shape and topology optimization are able to generate boundaries with satisfied smoothness and distinct interfaces. Moreover, the results (Fig 8) show that although the initial design had 17 holes, the optimized design has 13 holes, which shows that the proposed method can merge existing holes.

Table 2: Initial parameters and corresponding optimal solutions for Case (2.0)

\begin{tabular}{c|cccc}
\hline Case & $\left(\omega_{11}, \omega_{22}\right)$ & $\left(\eta_{1111}, \eta_{2222}, \eta_{1122}\right)$ & Objective properties & Optimal effective properties \\
\hline & & & $C^{O b j}=0.04\left[\begin{array}{ccc}1 & -0.5 & 0 \\
-0.5 & 1 & 0 \\
0 & 0 & 0\end{array}\right]$ & $C^{\mathrm{H}}=0.0616\left[\begin{array}{ccc}1 & -0.4821 \\
-0.4821 & 0 \\
1 & 0 \\
0 & 0 & 0.06819\end{array}\right]$ \\
$(2.0)$ & $(2.00,2.00)$ & $(0.05,0.05,1.00)$ & $\alpha^{o b j}=\left[\begin{array}{l}0 \\
0 \\
0\end{array}\right]$ & $\alpha^{\mathrm{H}}=\left[\begin{array}{c}0.0821 \\
0.0821 \\
0\end{array}\right]$ \\
\hline
\end{tabular}

To study the relationship between Poisson's ratio and thermal expansion coefficients, Case (2) is again used to design a balanced orthotropic metamaterial with the same negative Poisson's ratio of -0.5 but with different thermal expansion coefficients. In Case (2.1) a negative thermal strain coefficient in both directions is desired and in Case (2.2) a positive thermal strain coefficient is desired. The parameters and designs are given in Table 3. The results in the three cases show that there is no inherent relationship between Poisson's ratio and thermal expansion coefficients. The topologies for the total solid phases in Cases (2.0), (2.1) and (2.2) are similar, with very close Poisson's ratio values. However, different configurations for each solid 
material are generated, in order to achieve the different thermal expansion coefficients. The different responses to the same thermal load by the two different materials result in the deformation of the cellular structure when heated. Thus, how to configure solid materials is the main factor to determine the thermal expansion coefficients.

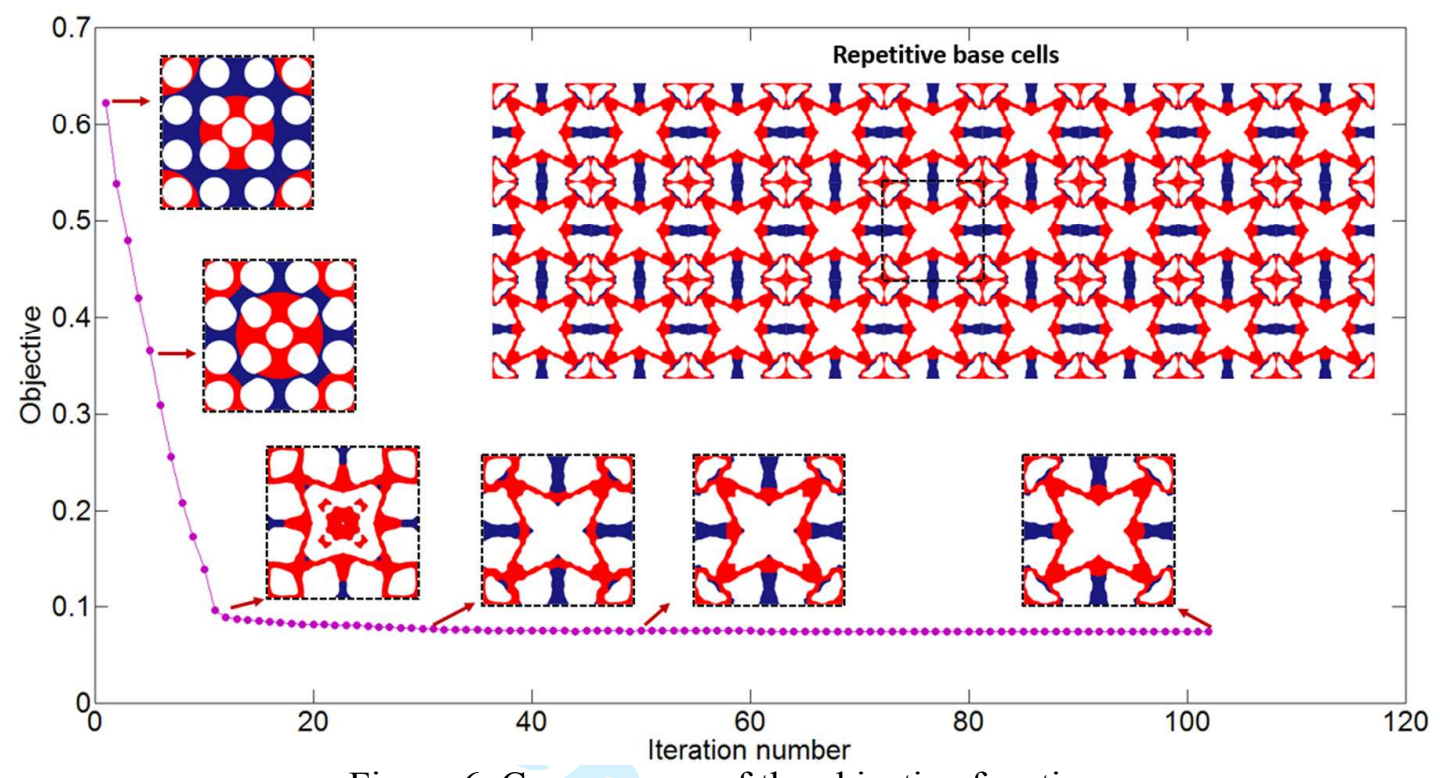

Figure 6. Convergence of the objective function

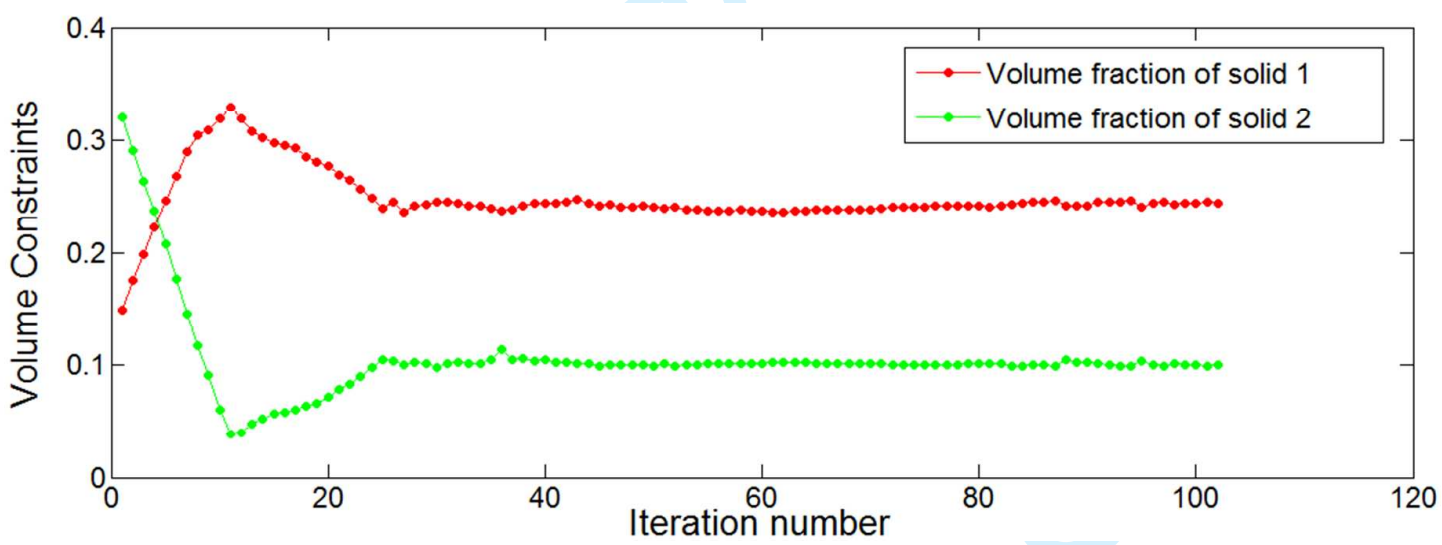

Figure 7. Convergence of two volume constraints

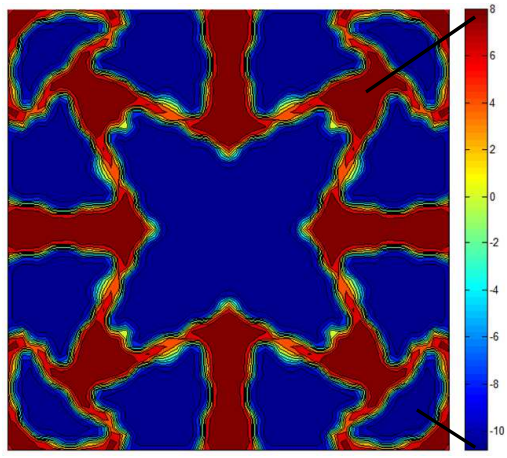

Figure 8. $\quad$ (a) Contours of LSF1 $\left(\Phi^{1}\right)$

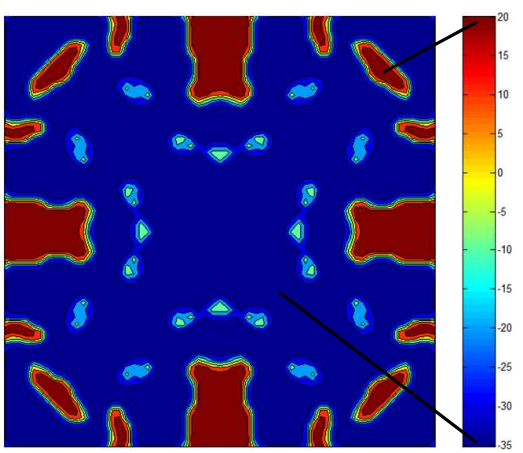

(b) Contours of LSF2 $\left(\Phi^{2}\right)$

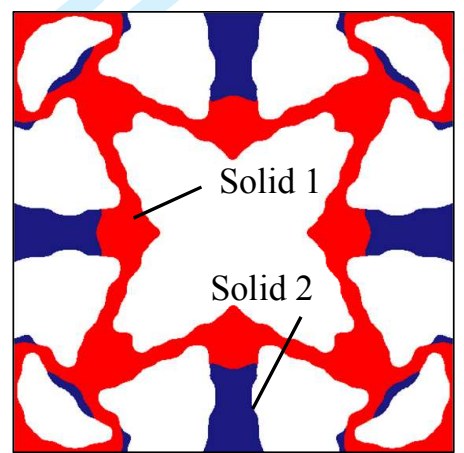

(c) Distribution of two solids: Solid $1\left(\Phi_{1}>0 \& \Phi_{2}<0\right.$, Red color online) and Solid $2\left(\Phi_{1}>0 \& \Phi_{2}>0\right.$, Blue color online) 


\section{Numerical Example 3}

Table 3: Initial parameters and corresponding optimal solutions for Cases (2.1) and (2.2)

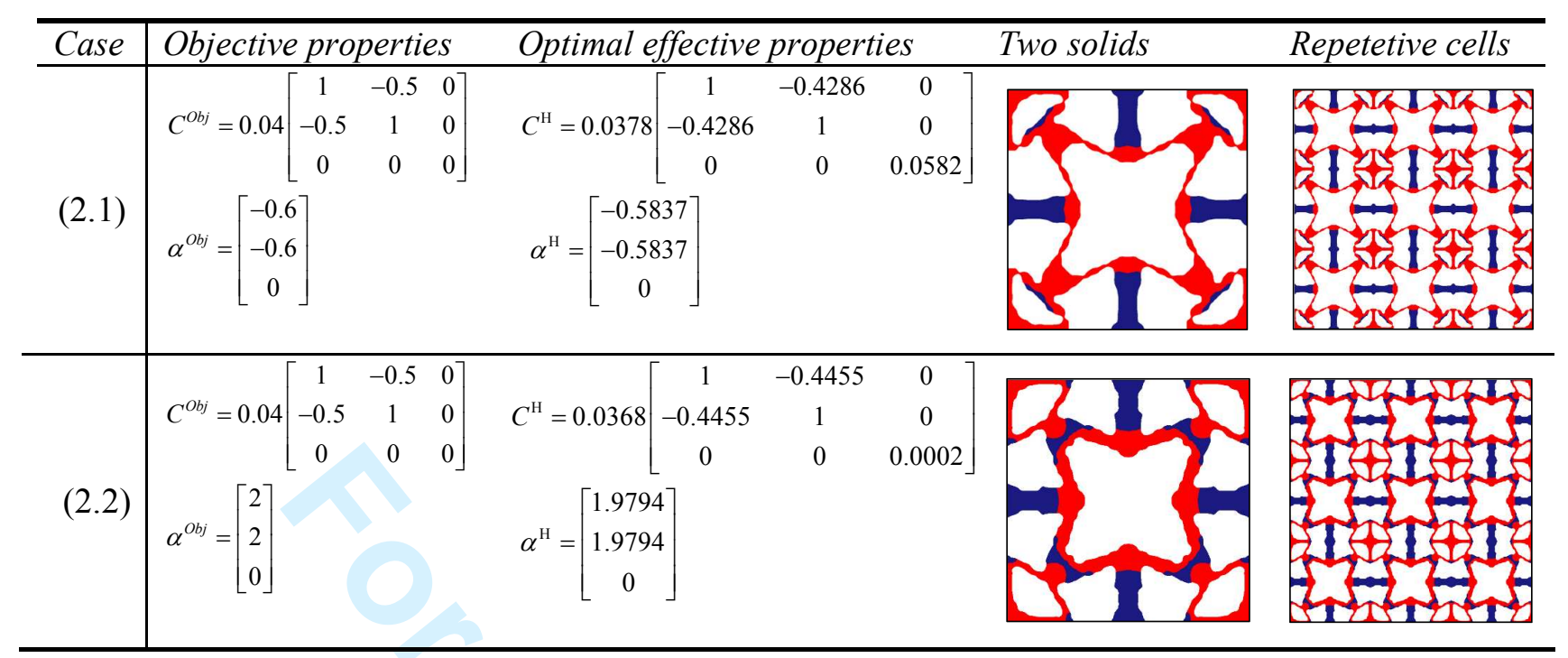

In Case (3), the objective is to design a balanced orthotropic (square symmetrical) metamaterial to achieve NPR and NTE simultaneously with two solid materials. The two artificial solid materials are assumed to have Young's moduli: $E^{1}=1, E^{2}=5$, Poisson's ratios: $v^{1}=0.3, v^{2}=0.5$, and thermal expansion coefficients $\alpha^{1}=1$ and $\alpha^{2}=10$. The desired negative Poisson's ratio is -0.5 and negative thermal expansion is -1 , in both directions. The horizontal and vertical geometric symmetries are included, and the volume constraints are $V$ ${ }^{1}=0.3$ and $0.15>=V^{2}>=0.1$, with other parameters given in Table 4. Optimization results are provided in Figs 9-12. The topologies obtained in Case (3) are similar to those in Cases (2.0-2.2), since the starting design was the same in all cases. However, different boundary shapes can be observed, and thinner bars and hingelike structures are generated in Case (3) to achieve smaller thermal strain than Cases (2.0-2.2). When selecting the parameters for initial design with two solid materials, the H-S bounds should be taken into account. It is known that the achieved effective properties are limited by the H-S bounds (Hashin and Shtrikman 1963).

Table 4: Initial parameters and corresponding optimal solutions for Case (3)

\begin{tabular}{l|ccccc}
\hline Case & $\left(\omega_{11}, \omega_{22}\right)$ & $\left(\eta_{1111}, \eta_{2222}, \eta_{1122}\right)$ & Objective properties & Optimal effective properties \\
\hline & & & $C^{O b j}=0.04\left[\begin{array}{ccc}1 & -0.5 & 0 \\
-0.5 & 1 & 0 \\
0 & 0 & 0\end{array}\right]$ & $C^{\mathrm{H}}=0.0435\left[\begin{array}{ccc}1 & -0.4345 & 0 \\
-0.4345 & 1 & 0 \\
0 & 0 & 0.0575\end{array}\right]$ \\
$(3)$ & $(2.00,2.00)$ & $(0.05,0.05,1.00)$ & $\alpha^{O b j}=\left[\begin{array}{c}-1 \\
-1 \\
0\end{array}\right]$ & $\alpha^{\mathrm{H}}=\left[\begin{array}{c}-0.9656 \\
-0.9656 \\
0\end{array}\right]$ \\
\hline
\end{tabular}

In the optimized result given in Fig. 11(c), it can be seen that there is a small ratio of soft materials (Solid 2) distributed over the surfaces at the locations of four corners of the hard solid material (Solid 1). The appearance of the soft materials is reasonable and no additional regularization technique is required to 
remove the small geometrical features. The soft materials around the four corners will facilitate the deformation and rotating effect of the microstructure. Although traditional manufacturing may not fabricate such microstructures, future developments in additive manufacturing may provide a way to resolve this issue. Methods to take advantage of additive manufacturing to fabricate such materials will be addressed in our ongoing research.

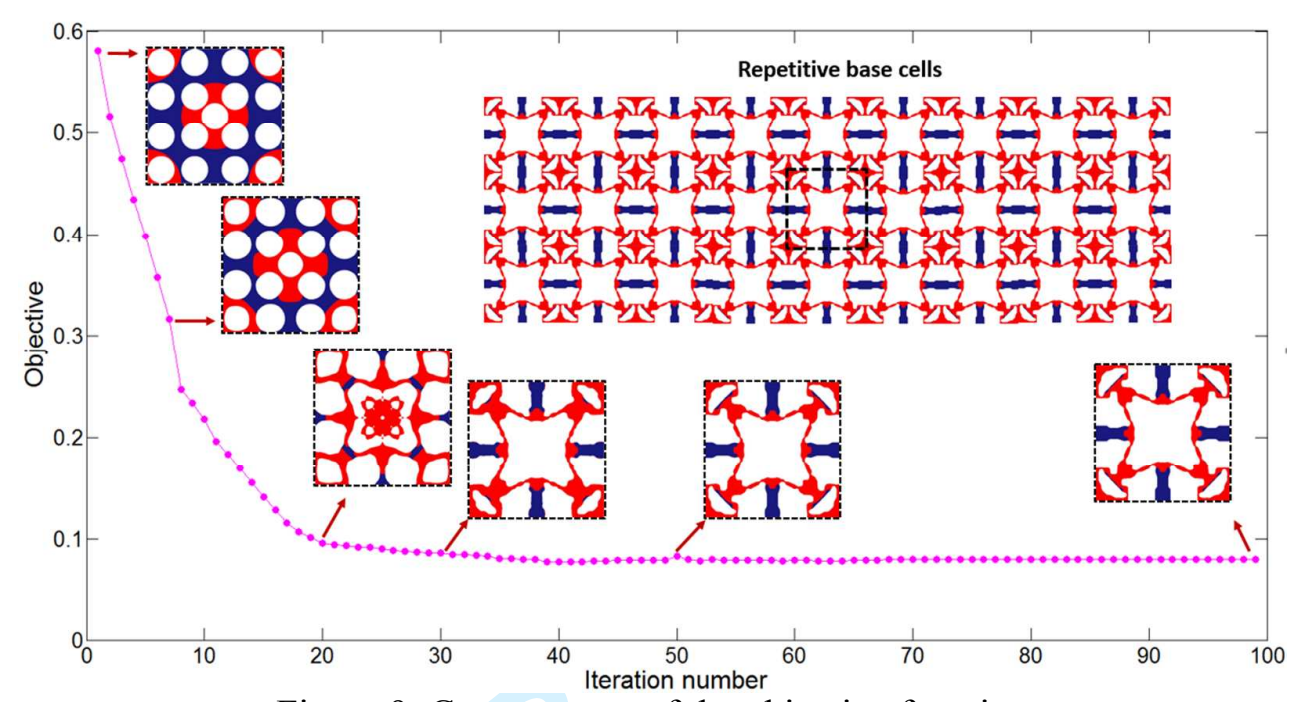

Figure 9: Convergence of the objective function

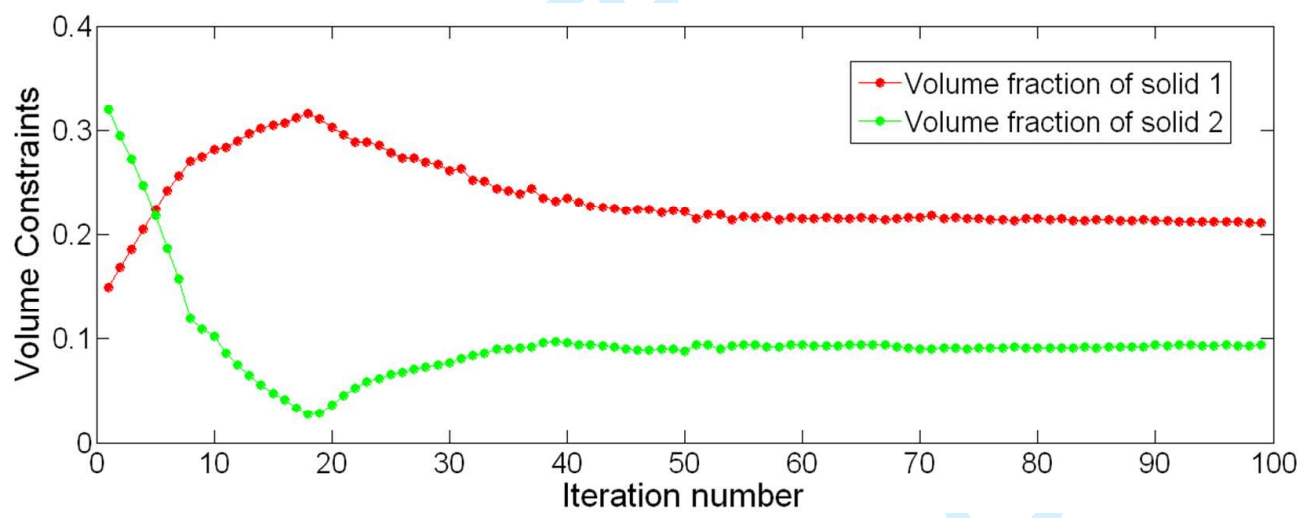

Figure 10. Convergence of two volume constraints

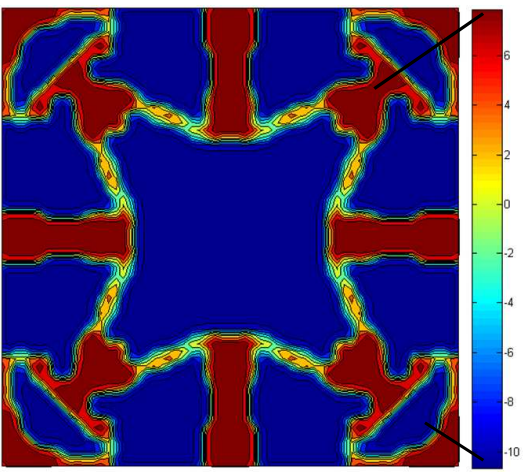

Figure 11.

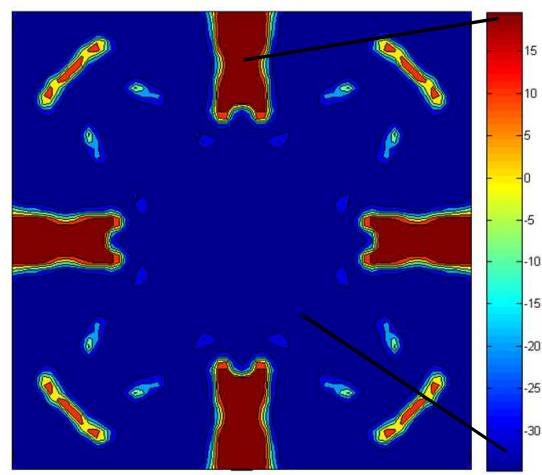

(b) Contours of LSF2 $\left(\Phi^{2}\right)$

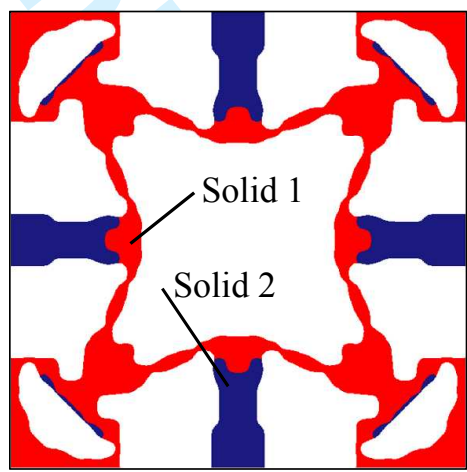

(c) Distribution of two solids: Solid $1\left(\Phi_{1}>0 \& \Phi_{2}<0\right.$, Red color online) and Solid 2 (Blue $\Phi_{1}>0 \& \Phi_{2}>0$, Blue color online) 


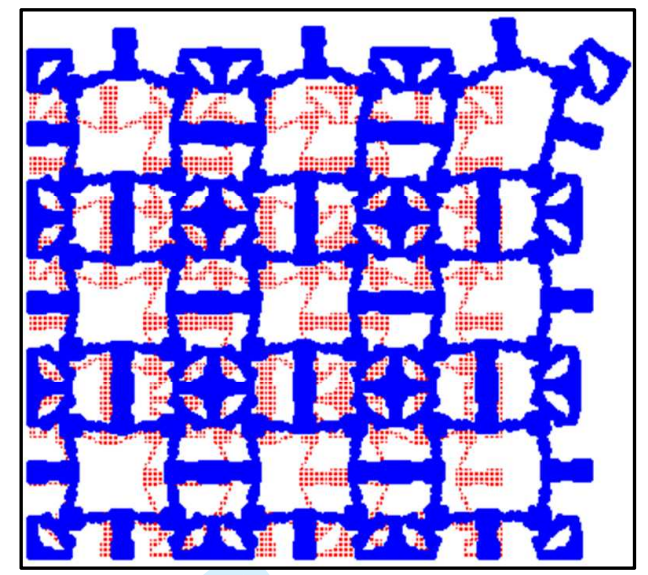

(a) Negative Poisson's ratio

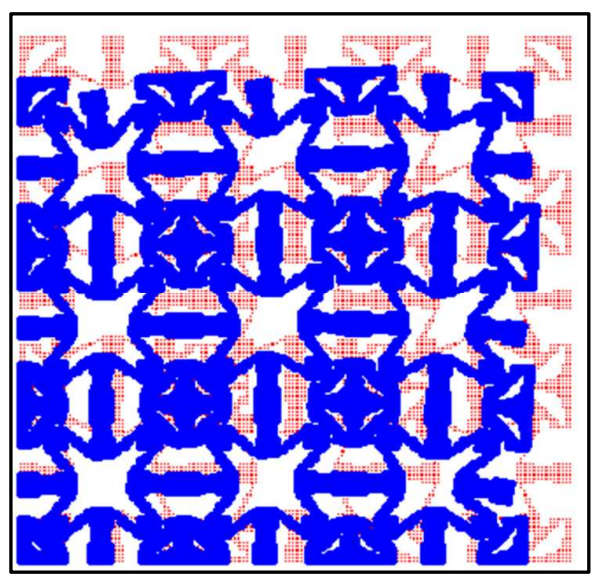

(b) Negative thermal expansion

Figure 12. Displacement plots of field nodes

\section{Conclusions}

This paper has proposed a computational design method for topology optimization of multifunctional mechanical metamaterials, in which the numerical homogenization method is used to evaluate the effective material properties, and a multiphase parametric level set method is applied to evolve shape and topology of the microstructure. The results of several numerical cases show the effectiveness of the proposed method for designing micro-structured metamaterials to have desired thermo-mechanical properties. This method may be extended to engineering other micro- and even nanostructured metamaterials. Selection of initial parameters is essential to achieve the optimized designs and ensure the stability of the optimization. To extend the proposed method to problems with more solid phases, additional numerical schemes may be required to overcome instability due to nonlinearity of the volume constraints.

Although there have as yet been not many particular applications for materials with both NPR and NTE, a mechanical metamaterial with double negative indexes is potentially useful. These kinds of multifunctional metamaterials exhibit thermoelastic behavior, which is impossible to obtain from conventional counterparts, which may provide opportunities for many novel and innovative material designs and applications.

\section{Acknowledgement}

This research is supported in part by the Australian Research Council-Discovery Project (DP160102491, DP150102751), the National Natural Science Foundation of China (51575204), and the Science and Technology Support Program of Hubei Province of China (2015BHE026).

\section{References}

Allaire, G. 2002. Shape Optimization by the Homogenization Method. New York: Springer. 
Allaire, G., F. Jouve, and A.-M. Toader. 2004. "Structural optimization using sensitivity analysis and a level-set method." Journal of Computational Physics 194(1): 363-393. doi:10.1016/j.jcp.2003.09.032.

Allaire, G., C. Dapogny, G. Delgado, and G. Michailidis. 2014. "Multi-phase structural optimization via a level set method." ESAIM - Control Optimisation and Calculus of Variations, EDP Sciences 20: 576-611.

Belytschko, T., S.P. Xiao, and C. Parimi. 2003. "Topology optimization with implicit functions and regularization.” International Journal for Numerical Methods in Engineering 57(8): 1177-1196. doi:10.1002/nme.824.

Bendsøe, M. P., and N. Kikuchi. 1988. "Generating optimal topologies in structural design using a homogenization method." Computer Methods in Applied Mechanics and Engineering 71(2): 197-224. doi:10.1016/00457825(88)90086-2.

Bendsøe, M.P., and O. Sigmund. 1999. "Material interpolation schemes in topology optimization." Archive of Applied Mechanics 69(9-10): 635-654. doi:10.1007/s004190050248.

Bendsøe, M. P., and O. Sigmund. 2003. Topology optimization: Theory, Methods and Applications. Berlin Heidelberg: Springer.

Buehler, M., B. Bettig, and G.G. Parker. 2004. "Topology optimization of smart structures using a homogenization approach." Journal of Intelligent Material Systems and Structures 15(8): 655-667. doi:10.1177/1045389X04043944.

Chen, H., and C. Chan. 2007. "Acoustic cloaking in three dimensions using acoustic metamaterials." Applied Physics Letters 91: 183518. doi:10.1063/1.2803315.

Cherkaev, A. 2000. Variational methods for structural optimization, New York: Springer.

Choi, K.K., and N.H. Kim. 2005. Structural sensitivity analysis and optimization I: linear systems. New York: Springer.

Dijk, N. P., K. Maute, M. Langelaar, and F. Keulen. 2013. "Level-set methods for structural topology optimization: a review." Structural and Multidisciplinary Optimization 48(3) 437-472. doi:10.1007/s00158-013-0912-y.

Evans, K. E., and A. Alderson. 2000. "Auxetic materials: functional materials and structures from lateral thinking." Advanced Materials 12(9): 617-628. doi:10.1002/(SICI)1521-4095(200005)12:9<617::AID-ADMA617>3.0.CO;2-3.

Gao, T., and W. Zhang. 2011. "A mass constraint formulation for structural topology optimization with multiphase materials." International Journal for Numerical Methods in Engineering 88(8): 774-796. doi:10.1002/nme.3197.

Gibiansky, L.V., and O. Sigmund. 2000. "Multiphase composites with extremal bulk modulus." Journal of the Mechanics and Physics of Solids 48(3) 461-498. doi:10.1016/S0022-5096(99)00043-5.

Grima, J.N., E. Chetcuti, E. Manicaro, D. Attard, M. Camilleri, R. Gatt, and K.E. Evans. 2012. "On the auxetic properties of generic rotating rigid triangles." Proceedings of the Royal Society A: Mathematical, Physical and Engineering Science 468(2319): 810-830. doi:10.1098/rspa.2011.0273.

Haber, E. 2004. “A multilevel, level-set method for optimizing eigenvalues in shape design problems.” Journal of Computational Physics 198(2): 518-534. doi:10.1016/j.jcp.2004.01.031.

Hashin, Z., and S. Shtrikman. 1963. "A variational approach to the theory of the elastic behaviour of multiphase materials." Journal of the Mechanics and Physics of Solids 11(2): 127-140. doi:10.1016/0022-5096(63)90060-7.

Kang, Z., and Y. Wang. 2011. "Structural topology optimization based on non-local Shepard interpolation of density field." Computer Methods in Applied Mechanics and Engineering 200(49-52): 3515-3525.

doi:10.1016/j.cma.2011.09.001.

Lakes, R. 1987. "Foam structures with a negative Poisson's ratio." Science 235(4792): 1038-1040.

doi:10.1126/science.235.4792.1038.

Lakes, R. 1996. “Cellular solid structures with unbounded thermal expansion.” Journal of Materials Science Letters 15(6): 475-477.

Lekhnitski1̌, S. G. 1963. "Theory of elasticity of an anisotropic elastic body.” San Francisco, California: Holden-Day.

L. Lu, T. Yamamoto, M. Otomori, T. Yamada, K. Izui, and S. Nishiwaki, S. 2013. "Topology optimization of an acoustic metamaterial with negative bulk modulus using local resonance." Finite Elements in Analysis and Design 72: 1-12. doi:10.1016/j.finel.2013.04.005. 
Luo, Z., L. Tong, M.Y. Wang, and S. Wang. 2007. "Shape and topology optimization of compliant mechanisms using a parameterization level set method." Journal of Computational Physics 227(1): 680-705. doi:10.1016/j.jcp.2007.08.011.

Luo, Z., M.Y. Wang, S. Wang, S. and P. Wei. 2008. "A level set-based parameterization method for structural shape and topology optimization." International Journal for Numerical Methods in Engineering 76(1): 1-26. doi:10.1002/nme.2092.

Luo, Z., L. Tong, P. Wei, and M.Y. Wang. 2009. "Design of piezoelectric actuators using a multiphase level set method of piecewise constants." Journal of Computational Physics 228(7): 2643-2659. doi:10.1016/j.jcp.2008.12.019.

Luo, Z., W. Gao, W., and C. Song. 2010. "Design of multi-phase piezoelectric actuators.” Journal of Intelligent Material Systems and Structures 21(18) 1851-1865. doi:10.1177/1045389X10389345.

Luo, Z., N. Zhang, W. Gao, and H. Ma. 2012. "Structural shape and topology optimization using a meshless Galerkin level set method." International Journal for Numerical Methods in Engineering 90(3): 369-389. doi:10.1002/nme.3325.

Luo, Z., N. Zhang, Y. Wang, and W. Gao. 2013. "Topology optimization of structures using meshless density variable approximants." International Journal for Numerical Methods in Engineering 93(4): 443-464. doi:10.1002/nme.4394.

Makhija, D., and K. Maute. 2014. "Numerical instabilities in level set topology optimization with the extended finite element method." Structural and Multidisciplinary Optimization 49(2) 185-197. doi:10.1007/s00158-013-0982-x.

Mei, Y., and X. Wang. 2004. "A level set method for structural topology optimization and its applications." Advances in Engineering Software 35(7): 415-441. doi:10.1016/j.advengsoft.2004.06.004.

Milton, G. W. 1992. "Composite materials with Poisson's ratios close to-1." Journal of the Mechanics and Physics of Solids 40(5): 1105-1137. doi:10.1016/0022-5096(92)90063-8.

Nicolaou, Z. G., and A. E. Motter. 2012. "Mechanical metamaterials with negative compressibility transitions." Nature Materials 11: 608-613. doi:10.1038/nmat3331.

Osher, S., and R. Fedkiw. 2003. Level set methods and dynamic implicit surfaces. New York: Springer.

Osher, S., and J.A. Sethian. 1988. "Fronts propagating with curvature-dependent speed: algorithms based on Hamilton-Jacobi formulations.” Journal of Computational Physics 79(1): 12-49. doi:10.1016/0021-9991(88)90002-2.

Otomori, M., T. Yamada, K. Izui, S. Nishiwaki, and J. Andkjær. 2012. "A topology optimization method based on the level set method for the design of negative permeability dielectric metamaterials." Computer Methods in Applied Mechanics and Engineering, 237-240: 192-211. doi:10.1016/j.cma.2012.04.022.

Scarpa, F., and G. Tomlinson. 2000. "Sandwich structures with negative Poisson's ratio for deployable structures." IUTAM-IASS Symposium on Deployable Structures: Theory and Applications 80: 335-343, Dordrecht: Springer.

Sethian, J. A. 1999. Level set methods and fast marching methods: evolving interfaces in computational geometry, fluid mechanics, computer vision, and materials science. Cambridge: Cambridge University Press.

Sethian, J. A., and A. Wiegmann. 2000. "Structural boundary design via level set and immersed interface methods." Journal of Computational Physics 163(2): 489-528. doi:10.1006/jcph.2000.6581.

Sigmund, O., and S. Torquato. 1996. "Composites with extremal thermal expansion coefficients." Applied Physics Letters 69(21): 3203-3205. doi:10.1063/1.117961.

Sigmund, O., and S. Torquato. 1997. "Design of materials with extreme thermal expansion using a three-phase topology optimization method." Journal of the Mechanics and Physics of Solids 45(6): 1037-1067.

doi:10.1016/S0022-5096(96)00114-7.

Sigmund, O. 2001. "Design of multiphysics actuators using topology optimization-Part I: One-material structures." Computer Methods in Applied Mechanics and Engineering 190(49-50) 6577-6604. doi:10.1016/S00457825(01)00251-1.

Smith, D., J. Pendry, and M. Wiltshire. 2004. "Metamaterials and negative refractive index." Science 305(5685) 788792. doi:10.1126/science.1096796.

Svanberg, K. 1987. "The method of moving asymptotes-a new method for structural optimization." International Journal for Numerical Methods in Engineering 24: 359-373. doi:10.1002/nme.1620240207. 
Tavakoli, R. 2014. "Multimaterial topology optimization by volume constrained Allen-Cahn system and regularized projected steepest descent method." Computer Methods in Applied Mechanics and Engineering 276: 534-565. doi:10.1016/j.cma.2014.04.005.

Tavakoli, R., and S.M. Mohseni. 2014. "Alternating active-phase algorithm for multimaterial topology optimization problems: a 115-line MATLAB implementation.” Structural and Multidisciplinary Optimization 49(4): 621-642. doi:10.1007/s00158-013-0999-1.

Veselago, V. G. 1968. "The electrodynamics of substances with simultaneously negative values of $\varepsilon$ and $\mu$. ." PhysicsUspekhi 10(4): 509-514. doi:10.1070/PU1968v010n04ABEH003699.

Wang, M. Y., X. Wang, and D. Guo. 2003. "A level set method for structural topology optimization.” Computer Methods in Applied Mechanics and Engineering 192(1-2): 227-246. doi:10.1016/S0045-7825(02)00559-5.

Wang, M.Y., and X. Wang. 2004. ““'Color” level sets: a multi-phase method for structural topology optimization with multiple materials." Computer Methods in Applied Mechanics and Engineering 193(6-8): 469-496. doi:10.1016/j.cma.2003.10.008.

Wang, M.Y., and X. Wang. 2005. "A level-set based variational method for design and optimization of heterogeneous objects." Computer-Aided Design 37(3): 321-337. doi:10.1016/j.cad.2004.03.007.

Wang, Y., Z. Luo, X. Zhang, and Z. Kang. 2014. "Topological design of compliant smart structures with embedded movable actuators." Smart Material Structures 23: 984-986. doi:http://dx.doi.org/10.1088/0964-1726/23/4/045024.

Wang, Y., Z. Luo, N. Zhang, and Z. Kang. 2014. "Topological shape optimization of microstructural metamaterials using a level set method.” Computational Materials Science 87: 178-186. doi:10.1016/j.commatsci.2014.02.006.

Wang, Y., Z. Luo, Z. Kang, and N. Zhang. 2015. "A multi-material level set-based topology and shape optimization method.” Computer Methods in Applied Mechanics and Engineering 283: 1570-1586. doi:10.1016/j.cma.2014.11.002.

Wei, P., and M.Y. Wang. 2009. "Piecewise constant level set method for structural topology optimization." International Journal for Numerical Methods in Engineering 78(4): 379-402. doi:10.1002/nme.2478.

Wendland, H. 2006. "Computational aspects of radial basis function approximation." Studies in Computational Mathematics 12: 231-256. doi:10.1016/S1570-579X(06)80010-8.

Xie, Y.M., and G.P. Steven. 1993. "A simple evolutionary procedure for structural optimization.” Computers \& Structures 49(5): 885-896. doi:10.1016/0045-7949(93)90035-C.

Yamada, T., K. Izui, S. Nishiwaki, and A. Takezawa. 2010. "A topology optimization method based on the level set method incorporating a fictitious interface energy." Computer Methods in Applied Mechanics and Engineering 199(45-48): 2876-2891. doi:10.1016/j.cma.2010.05.013.

Yang, W., Z. M. Li, W. Shi, B. H. Xie, and M. B. Yang. 2004. "Review on auxetic materials." Journal of Materials Science 39(10): 3269-3279.

Zhou, M., and G. I. N. Rozvany. 1991. "The COC algorithm, Part II: topological, geometrical and generalized shape optimization." Computer Methods in Applied Mechanics and Engineering 89(1-3): 309-336. doi:10.1016/00457825(91)90046-9.

Zhou, S., and M.Y. Wang. 2007. "Multimaterial structural topology optimization with a generalized Cahn-Hilliard model of multiphase transition." Structural \& Multidisciplinary Optimization 33: 89-111. doi:10.1007/s00158-0060035-9.

Zhou, S., W. Li, Y. Chen, G. Sun, and Q. Li. 2011. "Topology optimization for negative permeability metamaterials using level-set algorithm." Acta Materialia 59(7): 2624-2636. doi:10.1016/j.actamat.2010.12.049.

Zhu, J., W. Zhang, and P. Beckers. 2009. "Integrated layout design of multi-component system." International Journal for Numerical Methods in Engineering 78(6): 631-651. doi:10.1002/nme.2499. 